\title{
Chaos and the Shapes of Elliptical Galaxies
}

\author{
D. Merritt \\ Department of Physics and Astronomy, Rutgers University, New Brunswick, NJ 08855 \\ Rutgers Astrophysics Preprint Series No. 186 \\ To appear in SCIENCE, Jan. 19, 1996
}

\begin{abstract}
Hubble Space Telescope (HST) observations reveal that the density of stars in most elliptical galaxies rises toward the center in a power-law cusp. Many of these galaxies also contain central dark objects, possibly supermassive black holes. The gravitational force from a steep cusp or black hole will destroy most of the box orbits that constitute the "backbone" of a triaxial stellar system. Detailed modelling demonstrates that the resulting chaos can preclude a self-consistent, strongly triaxial equilibrium. Most elliptical galaxies may therefore be nearly axisymmetric, either oblate or prolate.
\end{abstract}

Information about the three-dimensional shape of a galaxy is lost when the galaxy is projected onto the plane of the sky. This loss of information is acute in the case of elliptical galaxies, whose apparent shapes are elliptical but whose intrinsic shapes could be oblate, prolate or fully triaxial.

Before about 1975, elliptical galaxies were thought to be rotationally flattened oblate spheroids. The discovery that elliptical galaxies rotate much more slowly than a fluid body with the same shape (1) led to the hypothesis that most of these systems are triaxial ellipsoids, with shapes that are maintained by anisotropic velocity dispersions rather than by centrifugal force (2). The triaxial hypothesis was supported by the successful construction of self-consistent triaxial models on the computer (3). Most of the stars in these numerical models occupied "regular" orbits that respect three isolating integrals, two in addition to the energy; the major families of regular orbits are the short- and long-axis "tubes," and the "boxes" (4). Box orbits are uniquely associated with the triaxial geometry; they densely fill a box- or bowtie-shaped region, and a star on such an orbit passes arbitrarily close to the galaxy center after many oscillations. The time-averaged shape of a box orbit mimics that of the underlying galaxy, and the potential from a star on a box orbit helps support 
the triaxial shape of the galaxy as a whole. Box orbits are always found to be strongly populated in the self-consistent triaxial models.

Box orbits exist only in triaxial potentials with "cores," that is, models in which the density near the center is approximately constant and the corresponding gravitational potential is roughly quadratic in the coordinates (5). Recent HST observations of the centers of elliptical galaxies (6) reveal that these galaxies almost never have constant-density cores; the stellar density always continues to rise, roughly as a power law, into the smallest observable radius. In fainter ellipticals, the stellar density increases roughly as $\rho \propto r^{-2}$, while for brighter ellipticals the cusp slope is $\rho \propto r^{-1}$ or shallower (7). In addition, there is increasingly strong evidence for massive dark objects (MDO's), possibly supermassive black holes, at the centers of many elliptical galaxies (8). In the most convincing cases, these central singularities appear to contain as much as $1 \%$ of the total mass of the galaxy.

A central mass concentration can strongly perturb the motion of a star on a box orbit, regardless of its apocenter distance, since a star on such an orbit will eventually pass arbitrarily close to the center and be deflected by the strong gravitational force there (9). The result is a sensitive dependence of the orbital trajectory on initial conditions; in other words, the orbit loses its two non-classical integrals of motion and becomes chaotic. The degree of chaos can be quantified via the Liapunov characteristic numbers that measure the average rate of exponential divergence of two initially nearby trajectories. Fig. 1a shows histograms of Liapunov numbers for ensembles of box-like orbits (defined as orbits that have a stationary point) at one energy in a triaxial model with the density law

$$
\rho(m)=\rho_{0}\left(m^{2}+m_{0}^{2}\right)^{-1}\left(1+m^{2}\right)^{-1}, \quad m^{2}=\frac{x^{2}}{a^{2}}+\frac{y^{2}}{b^{2}}+\frac{z^{2}}{c^{2}},
$$

with $c / a=0.5$ and $b / a=0.79$. The parameter $m_{0}$ is a "core radius"; when $m_{0}=1$, Eq. 1 reduces to the "Perfect Ellipsoid" in which all orbits are regular (10), while for small $m_{0}$, this density law has an $r^{-2}$ central cusp similar to those observed in many elliptical galaxies.

When $m_{0}<10^{-1}$, one finds that most of the box-like orbits are chaotic; the only exceptions are orbits which lie close to stable, resonant orbits that avoid the center (Fig. 2a). The destruction of the box orbits also occurs in models with a central singularity, or "black hole" (Fig. 1b, 2b). In both cases, the typical Liapunov time scale for divergence of nearby trajectories is 3-5 times the oscillation period of the long-axis closed orbit of the same energy, henceforth defined as the "dynamical time" (Table 1). Stars in the central regions of elliptical galaxies have made $10^{2}-10^{3}$ radial oscillations since the epoch of galaxy formation; thus, elliptical galaxies are many Liapunov times old.

A star on a chaotic orbit will eventually visit every point in configuration space consistent with energy conservation; it will fill a region inside of the equipotential surface 
corresponding to its energy. These surfaces are more nearly spherical than the equidensity surfaces that define the galaxy figure; hence, chaotic orbits are less useful than regular box orbits for building self-consistent models. However, even chaotic orbits have structure. Fig. 3 illustrates the density of an ensemble of stars that fills chaotic phase space in an approximately time-independent way at one energy. The shape is similar to that of a superposition of box-like orbits, since the chaotic trajectory fills that part of phase space that would have been occupied by box orbits in a fully integrable model. But because the chaotic phase space at a given energy is interconnected via the "Arnold web" (11), there exists just a single invariant density like the one shown in Fig. 3 at each energy.

The "mixing time" of a chaotic orbit may be defined as the time required for an ensemble of stars on that orbit to reach a fully-mixed state like that of Fig. 3. In a real galaxy, the mixing process is likely to be extremely complex, involving violent collapse and rapidly varying forces during galaxy formation (12). However we can place an upper limit on the mixing time by asking how long it takes an ensemble of stars in the chaotic phase space of a time-independent potential to fill its allowed phase-space region in a nearly uniform way. Numerical experiments show that this relaxation process is roughly exponential, with a time constant of about 100 dynamical times in triaxial potentials with steep cusps or massive central singularities (13). After a few hundred dynamical times, the density in the chaotic phase-space region achieves a nearly constant, coarse-grained value and ceases to evolve. We would therefore expect the chaotic orbits in at least the central regions of a triaxial galaxy with a strong central mass concentration to be fully mixed.

The invariant distribution of Fig. 3 plays the role of a single orbit: it represents an unchanging and irreducible distribution of stars and can be used as a building block for a self-consistent model. However - unlike the regular orbits, which respect three integrals of the motion and therefore comprise a two-parameter family at every energy - there is only one invariant density at each energy in chaotic phase space. The replacement of the regular box orbits by chaotic trajectories thus limits the freedom to construct a self-consistent model, since it effectively reduces the number of different orbits. This limitation does not exist in oblate or prolate geometries, however, since axisymmetric potentials support only tube orbits, all of which avoid the center and most of which remain regular. Thus the nonexistence of a triaxial equilibrium with a given density profile would imply that a galaxy with the same mass distribution must be either axisymmetric, or in the process of evolving toward an axisymmetric state.

The degree to which chaos limits the freedom to construct triaxial equilibria was explored via two models with Dehnen's (14) density law:

$$
\rho(m)=\rho_{0} m^{-\gamma}(1+m)^{-(4-\gamma)} .
$$


The first model explored (the "strong cusp" model) had $\gamma \approx 2$, corresponding to fainter elliptical galaxies like M32. The "weak cusp" model had $\gamma \approx 1$, a good description of brighter ellipticals like M87. The axis ratios were chosen to be $c / a=0.5$ and $b / a=0.79$. A total of 7000 orbits were integrated for 100 dynamical times in each of the models, and their time-averaged densities stored in a grid of $10^{3}$ cells. A quadratic-programming algorithm was then used to find a set of non-negative orbital weights that reproduced the known mass of the model in the cells (15).

Attempts to construct self-consistent solutions using just the regular orbits failed for both mass models. Quasi-equilibrium solutions - in which chaotic orbits, computed for just 100 orbital periods, were included with arbitrary orbital weights - were found to exist for both weak- and strong-cusp models. However, real galaxies constructed in this way would evolve near the center as the chaotic orbits mixed toward their invariant distributions at each energy (16).

More nearly stationary solutions were successfully constructed for the weak-cusp mass model; all of the chaotic orbits within the inner half-mass radius could be replaced by the smaller set of invariant distributions without violating self-consistency. However these models could not be made fully mixed at both large and small radii. No significant fraction of the mass could be placed on fully-mixed chaotic orbits in the strong-cusp model without driving the solution away from self-consistency. The greater freedom to find solutions in the weak-cusp case resulted from the larger number of regular orbit families in this potential, which allowed less weight to be placed on the chaotic orbits.

These attempts to find self-consistent equilbria were based on only two, strongly triaxial mass models; more nearly axisymmetric models with the same density profile would presumably be easier to construct. However these results demonstrate that chaos can severely reduce the size of solution space for triaxial models and, at least in some cases, preclude self-consistent equilibria. It is reasonable to conclude that strongly triaxial configurations are difficult to construct in a self-consistent way, and that real ellipticals are either axisymmetric or nearly axisymmetric.

Although no attempts have yet been made to construct self-consistent triaxial models with central "black holes," the results shown here in Figs. 1 and 2 suggest that chaos would constrain such solutions about as strongly as it constrains triaxial models with steep density cusps. Secure detections of MDO's have been made in at least two elliptical galaxies $(17,18)$; the kinematical signature in both cases was a high streaming velocity of stars or gas very near the center. In addition, strong kinematical evidence for MDO's has been found in a number of S0 galaxies and spirals with bulges (8). The failure to detect MDO's in some ellipticals may be due to the lack of a rotating subpopulation, or to the fact that 
the galaxy is not oriented in such a way that the rotation is easily observed. The average mass of the MDO's in these three galaxies is $\sim 0.0045$ when expressed in units of the total stellar mass of the galaxy. This is close to the average mass required per galaxy if MDO's are dead quasars (19), which suggests that most or all ellipticals may harbor nuclear black holes.

If most elliptical galaxies contain steep density cusps, nuclear black holes, or both, then the arguments given above suggest that axisymmetry would generally be preferred over triaxiality for these galaxies. This would be especially true for lower-luminosity ellipticals which have the steepest cusps and the shortest dynamical times on average (6). The axisymmetric hypothesis is difficult to confirm since no unambiguous test for triaxiality exists. However, most recent studies of elliptical galaxy intrinsic shapes have found that few if any elliptical galaxies need to be strongly triaxial (20).

The dependence of observed rotation rate on flattening in low-luminosity ellipticals has long been known to be consistent with oblate symmetry for these galaxies (21). A classical test for triaxiality is dependence of major-axis orientation on radius (22). Such "isophote twists" are seen in a number of elliptical galaxies, but their interpretation is complicated by the likelihood that some of the twisted galaxies are not relaxed, while in others the twist may result from misaligned disks and bars. A stronger test for triaxiality is the detection of stellar streaming along the apparent minor axis of a galaxy (22). Minor axis rotation is rare, however, and a statistical study of the 38 elliptical galaxies for which two-dimensional velocity data are available suggests that the data can be well fit by a distribution in which $60 \%$ of galaxies are oblate and $40 \%$ prolate $(23)$.

The shapes of a few elliptical galaxies have been constrained by detailed comparison of numerical models with kinematical data. The best example is M32, the dwarf companion to the Andromeda galaxy. M32 has a $\rho \propto r^{-1.6}$ stellar cusp and also shows convincing evidence for a MDO containing $\sim 0.25 \%$ of the total galaxy mass (17); thus one would expect axisymmetry to be strongly preferred for this galaxy. In fact, oblate models reproduce the detailed kinematics of M32 extremely well (24). Rings and disks of gas or dust can sometimes be used as tracers of the shape of the gravitational potential in elliptical galaxies (25), although few of these subsystems are both extended and regular enough that the results are convincing. However the kinematics of a neutral hydrogen ring surrounding the elliptical galaxy IC 2006 suggests that its dark halo is accurately axisymmetric (26).

Bright ellipticals are observed to be slowly rotating, and if these galaxies are generically axisymmetric, their slow rotation implies nearly equal numbers of stars on tube orbits travelling in both directions. Such a configuration would arise naturally as the potential evolved from triaxiality into axial symmetry via the mechanism described here: stars on 
box-like orbits undergo periodic changes in the direction of their angular momenta, and an ensemble of such stars would presumably end up populating a set of tube orbits with roughly equal numbers of rotating and counter-rotating members. A galaxy with this orbital composition might reveal itself via a strongly flattened or double-peaked distribution of line-of-sight velocities (27). 


\section{REFERENCES}

1. F. Bertola and M. Capaccioli, Astrophys. J. 200, 439 (1975).

2. J. J. Binney, Mon. Not. R. Astron. Soc. 183, 501 (1978).

3. M. Schwarzschild, Astrophys. J. 232, 236 (1979).

4. An illustration of the major orbit families in integrable triaxial models may be found in D. Merritt, Science 259, 1867 (1993).

5. J. Lees and M. Schwarzschild, Astrophys. J. 384, 491 (1992).

6. Kormendy, J. et al., in ESO/OHP Workshop on Dwarf Galaxies, G. Meylan and P. Prugniel, Eds. (European Southern Observatory, Garching, 1995), p. 147.

7. D. Merritt and T. Fridman, in ASP Conf. Ser. Vol. 50, Fresh Views of Elliptical Galaxies, A. Buzzoni, A. Renzini and A. Serrano, Eds. (Astronomical Society of the Pacific, San Francisco, 1995), p. 13.

8. J. Kormendy and D. Richstone, Annual Review of Astronomy and Astrophysics, 33 (1995).

9. O. E. Gerhard and J. J. Binney, Mon. Not. R. Astron. Soc. 216, 467 (1985).

10. G. G. Kuzmin, in Dynamics of Galaxies and Clusters, T. B. Omarov, Ed. (Akademie Nauk Kazakh SSR, Alma Ata, 1973), p. 71; P. T. de Zeeuw and D. Lynden-Bell, Mon. Not. R. Astron. Soc. 215, 713, (1985).

11. A. J. Lichtenberg and M. A. Lieberman, Regular and Stochastic Motion (Springer-Verlag, New York, 1989).

12. D. Lynden-Bell, Mon. Not. R. Astron. Soc. 136, 101 (1967).

13. D. Merritt and M. Valluri, in preparation.

14. W. Dehnen, Mon. Not. R. Astron. Soc. 265, 250 (1993).

15. D. Merritt and T. Fridman, Astrophys. J. 456 (1996).

16. M. Schwarzschild, Astrophys. J. 409, 563 (1993).

17. A. Dressler and D. Richstone, Astrophys. J. 324, 701 (1988); van der Marel, R. P., de Zeeuw, T., Rix, H.-W., White, S. D. M., Mon. Not. R. Astron. Soc. 271, 99.

18. R. J. Harms et al., Astrophys. J. 435, L35 (1994). 
19. A. Soltan, Mon. Not. R. Astron. Soc. 200, 115 (1982).

20. D. Merritt, in Morphological and Physical Classification of Galaxies, G. Longo, M. Capaccioli, G. Busarello, Eds. (Kluwer Academic, Norwell, MA, 1992), pp. 309-320.

21. R. L. Davies, G. Efstathiou, S. M. Fall, G. Illingworth, and P. L. Schechter, Astrophys. J. 266, 41 (1983).

22. G. Contopoulos, Zeitschrift für Astrophysik 39, 126 (1956).

23. M. Franx, G. D. Illingworth and P. T. de Zeeuw, Astrophys. J. 383, 112 (1991).

24. E. E. Qian, P. T. de Zeeuw, and C. Hunter, Mon. Not. R. Astron. Soc. 274, 602 (1995).

25. F. Bertola et al., Astrophys. J. 373, 369 (1991).

26. M. Franx and T. de Zeeuw, Astrophys. J. Letters 392, L47 (1992).

27. O. Gerhard, Mon. Not. R. Astron. Soc. 265, 213 (1993).

28. M. Valluri carried out the calculations on which Fig. 3 was based, and made helpful comments on the manuscript. S. Tremaine, as referee, also made a number of suggestions that improved the presentation. This work was supported by NSF grant AST 93-18617 and by NASA grant NAG 5-2803. 
Table 1: Liapunov numbers of boxlike orbits in triaxial potentials. Orbits were computed for $10^{4}$ dynamical times at the half-mass energy in the potential corresponding to Eq. 1, with $c / a=0.5$ and $b / a=0.79$. Liapunov numbers $\sigma_{1}$ and $\sigma_{2}$ are given in units of the dynamical time; $M_{B H}$ represents black hole mass in units of the total mass of the model.

\begin{tabular}{rlcc}
$m_{0}$ & $M_{B H}$ & $\sigma_{1}$ & $\sigma_{2}$ \\
\hline $10^{-1}$ & - & $0.14 \pm 0.06$ & $0.045 \pm 0.02$ \\
$10^{-2}$ & - & $0.21 \pm 0.09$ & $0.078 \pm 0.03$ \\
$10^{-3}$ & - & $0.27 \pm 0.05$ & $0.085 \pm 0.02$ \\
$10^{-1}$ & $10^{-3}$ & $0.15 \pm 0.03$ & $0.066 \pm 0.02$ \\
$10^{-1}$ & $3 \times 10^{-3}$ & $0.20 \pm 0.04$ & $0.097 \pm 0.02$ \\
$10^{-1}$ & $10^{-2}$ & $0.28 \pm 0.08$ & $0.16 \pm 0.04$ \\
\hline
\end{tabular}


Fig. 1.- Histograms of Liapunov numbers for iso-energetic ensembles of box-like orbits in triaxial potentials. Starting points for the orbits were chosen from a uniform grid on an equipotential surface near the half-mass radius of the model (Fig. 2). Each orbit was integrated for $10^{4}$ dynamical times $T_{d}$; the thick curve represents the largest Liapunov number $\sigma_{1}$, the thin curve the second Liapunov number $\sigma_{2}$. Regular orbits lie in the narrow peaks near $\sigma T_{d}=0$. (a) $m_{0}=10^{-3}$; (b) $m_{0}=10^{-1}$, and a central point mass containing $0.3 \%$ of the total galaxy mass has been added.

Fig. 2.- Starting points of the regular and chaotic orbits whose Liapunov numbers make up the histograms of Fig. 1a and 1b. Each dot represents an initial point on one octant of the equipotential surface; small dots are chaotic orbits and large dots are regular orbits. Every orbit was dropped with zero velocity from this surface. The $X[Z]$ axes are the long [short] axes of the figure.

Fig. 3.- Fig. 3. Invariant density of an isoenergetic ensemble of 5000 stars in the chaotic phase space of a triaxial model with $m_{0}=10^{-3}, c / a=0.5$ and $b / a=0.79$. The $X[Z]$ axis is the long [short] axis of the triaxial figure. Plotted are the densities near each of the three principal planes. 

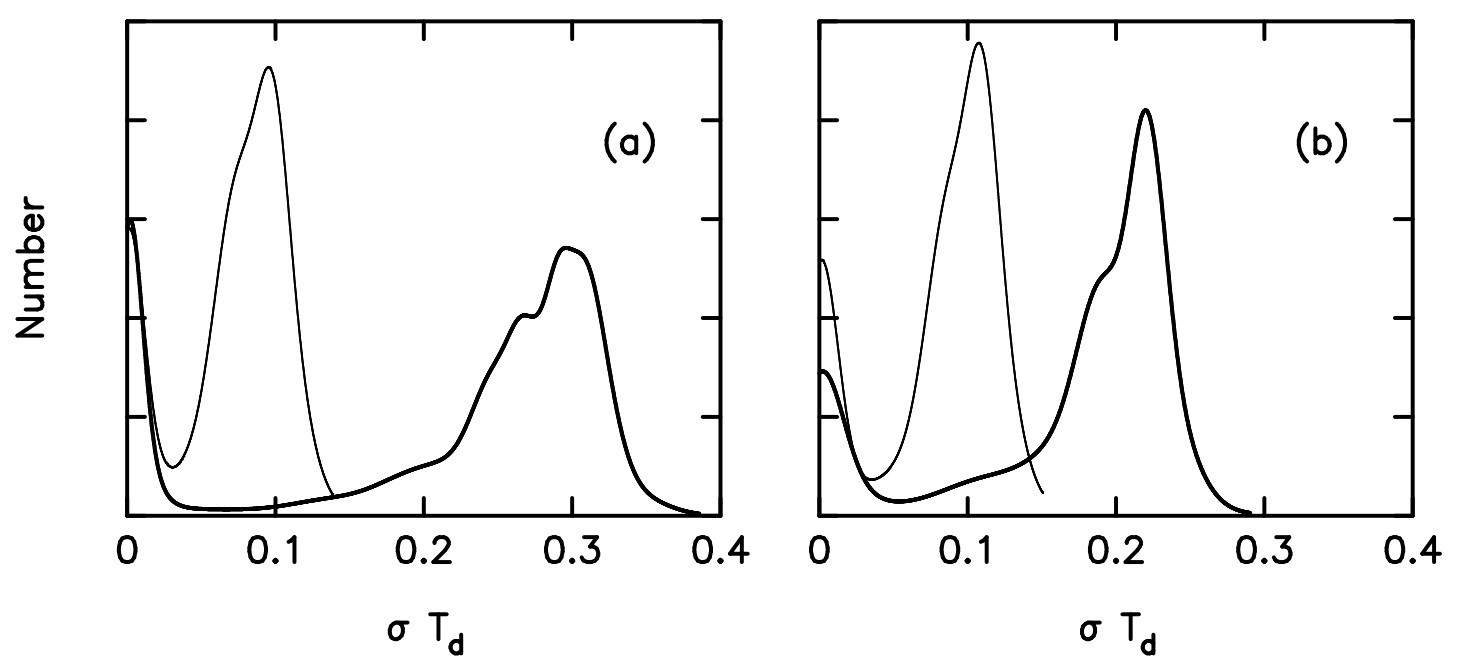

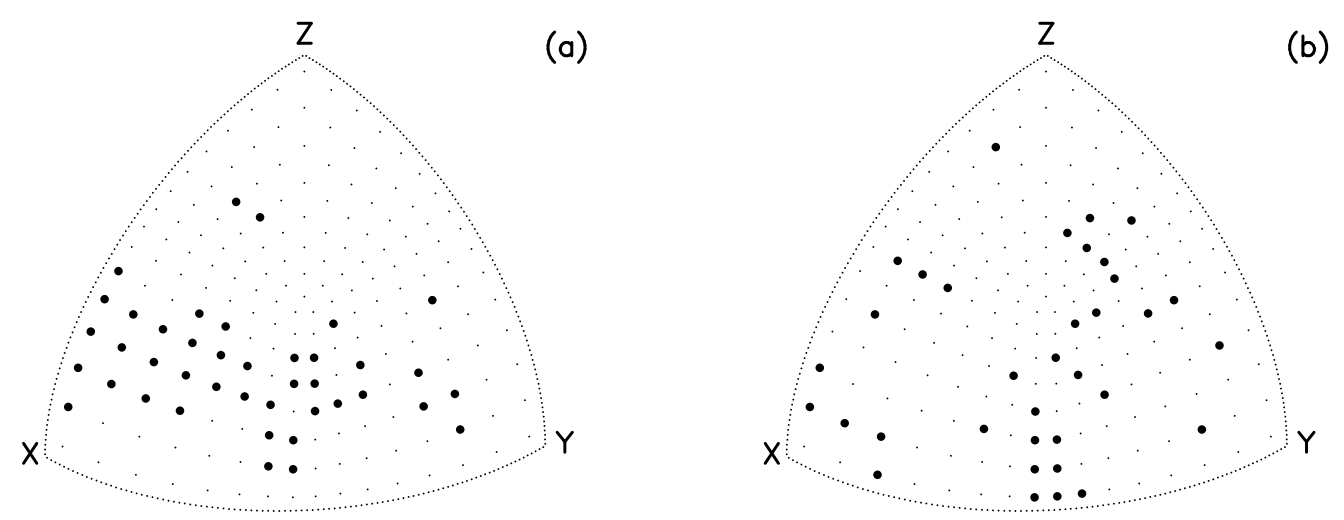


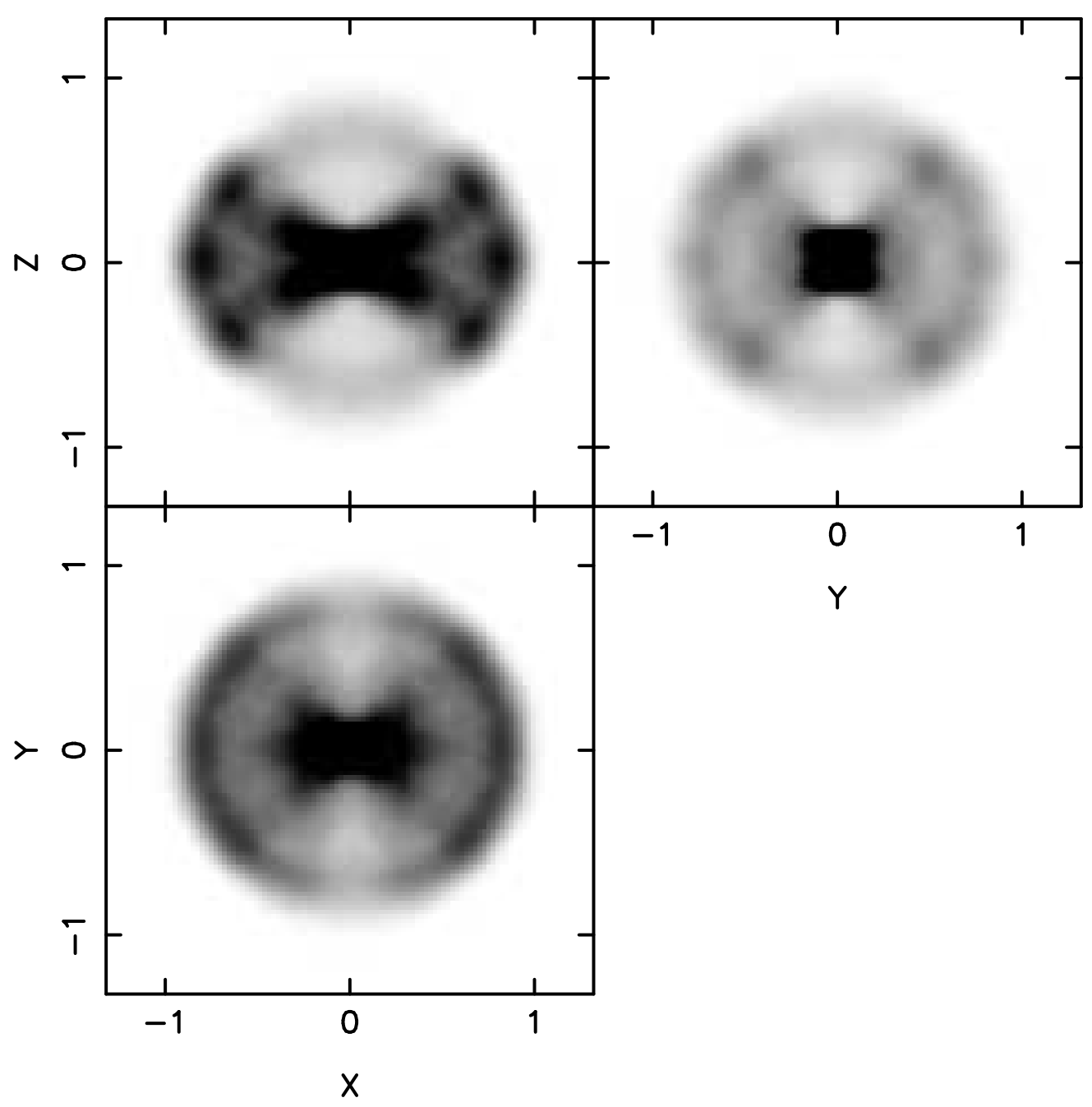

